\title{
Social uncertainty is heterogeneous and sometimes valuable
}

\author{
To win friends, help the needy, avoid exploitation or influence strangers, people must make decisions that are \\ inherently uncertain. In their compelling and insightful perspective on resolving social uncertainty', FeldmanHall \\ and Shenhav (henceforth F\&S) join a growing movement combining computational approaches with social \\ psychological theory ${ }^{2}$. F\&S identify a range of negative and positive aspects of social uncertainty. Here we offer \\ additional ways to think about social uncertainty and suggest potential avenues for future research.
}

F\&S define social uncertainty as the "degree to which a person's uncertainty about [...] their own future states and actions depends on their uncertainty about the states and actions of others." This is surely a central source of uncertainty in social life; further ground can be covered by distinguishing varieties of uncertainty that differentially influence social behaviour. For example, people are more selfish when they are uncertain about what outcomes their decisions will produce for others ${ }^{3}$, but less selfish when they are uncertain about the impact of those outcomes on others' welfare ${ }^{4}$. People also intuitively distinguish between epistemic uncertainty, which is resolvable with additional information, and aleatory uncertainty, which arises from randomness and is not resolvable with additional information ${ }^{5}$. It remains to be seen whether the unresolvable uncertainty arising from the inherent opacity of other minds is a special form of aleatory uncertainty or an entirely different species. Regardless, it is already clear that uncertainty does not uniformly affect social interactions.

In describing how people resolve social uncertainty, F\&S draw on a Bayesian framework that accurately characterizes not just predictions of others' behaviors ${ }^{6-8}$, but also people's global impressions about others' competence and moral character ${ }^{8}$. Bayesian inference provides a benchmark for establishing whether belief updating is 'optimal' in an information-theoretic sense. Systematic deviations from Bayes optimality could indicate social biases arising from heuristics ${ }^{1}$, reveal maladaptive social psychopathologies ${ }^{1,9}$ or even identify cognitive strategies that are optimal in an ecological sense-for example, maintaining uncertainty about the moral character of badly behaving others, which can help preserve relationships in case initial bad impressions turn out to be mistaken ${ }^{8}$.

Finally, F\&S identify several positive consequences of reducing social uncertainty, such as maintaining internal consistency and preserving a social identity (Box 1). In addition, their perspective raises the intriguing question of when social uncertainty itself is experienced as positive. Much of social life involves trading off costs and benefits for oneself and others, and robust evidence shows that when people face such trade-offs, they can find uncertainty attractive rather than aversive ${ }^{3,4}$. When uncertainty conceals how self-serving decisions will affect others, people exploit this 'moral wiggle room' to behave selfishly ${ }^{3,4,10}$ and prefer not to resolve uncertainty, even when doing so costs nothing ${ }^{3,4}$. In this way, uncertainty helps people preserve their moral self-image.

We applaud F\&S for advocating a productive and powerful inquiry into the computational substrates of social uncertainty. The challenge for future research will be to incorporate multiple varieties of uncertainty into models of social inference; to better characterize when social inference departs from the Bayesian ideal; and to develop new models that can illuminate when uncertainty is something people wish to avoid versus embrace.

Andreas Kappes (D)1,

Anne-Marie Nussberger (D),

Jenifer Z. Siegel (D)2, Robb B. Rutledge (D)3 and Molly J. Crockett (D) $4 *$

${ }^{1}$ Department of Psychology, City University London, London, UK. ${ }^{2}$ Department of Experimental Psychology, University of Oxford, Oxford, UK. ${ }^{3}$ Max Planck Centre for Computational Psychiatry and Ageing, University College London, London, UK. ${ }^{4}$ Department of Psychology, Yale University, New Haven, CT, USA.

*e-mail:molly.crockett@yale.edu

Published online: 29 July 2019

https://doi.org/10.1038/s41562-019-0662-y

References

1. FeldmanHall, O. \& Shenhav, A. Nat. Hum. Behav. 3, 426-435 (2019).

2. Cushman, F. \& Gershman, S. Top. Cogn. Sci. 11, 281-298 (2019).

3. Dana, J., Weber, R. A. \& Kuang, J. X. Econ. Theory 33, 67-80 (2007).

4. Kappes, A. et al. Nat. Hum. Behav. 2, 573-580 (2018).

5. Ülkümen, G., Fox, C. R. \& Malle, B. F. J. Exp. Psychol. Gen. 145, 1280-1297 (2016).

6. Behrens, T. E. J., Hunt, L. T., Woolrich, M. W. \& Rushworth, M. F. S. Nature 456, 245-249 (2008).

7. Diaconescu, A. O. et al. PLoS Comput. Biol. 10, e1003810 (2014).

8. Siegel, J. Z. et al. Nat. Hum. Behav. 2, 750-756 (2018).

9. Huys, Q. J., Guitart-Masip, M., Dolan, R. J. \& Dayan, P. Clin. Psychol. Sci. 3, 400-421 (2015).

10. Haisley, E. C. \& Weber, R. A. Games Econ. Behav. 68, 614-625 (2010).

Competing interests

The authors declare no competing interests. 